\title{
Dimensions of Knowledge and Ways of Thinking of Irrational Numbers
}

\author{
By Nina Hayfa* \\ Layal Saikaly ${ }^{\dagger}$
}

This paper explores the dimensions of knowledge and the ways of thinking (procedural or proceptual thinking) adopted by 157 Lebanese students in different grades when defining and identifying irrational numbers when locating them on the number line. It searches for a relation between the performance of the participants and their different grades. The results show the participants' inconsistency in the dimensions of knowledge used and a dependency on the procedural ways of thinking is detected. A change in the performance within different grades is also found except when identifying the nature of the given numbers.

Keywords: dimensions of knowledge, irrational numbers, procedural and proceptual thinking

\section{Introduction}

Being essential to the completeness of the set of real numbers, irrational numbers are defined by Dedekind (1901) as the cut which is created in order to fill the gaps produced by rational numbers. In this report, participants are expected to give one of two definitions, either as the numbers that cannot be written as a ratio of two integers $a$ and $b(b \neq 0)$, or as the numbers with an unlimited, un-periodic decimal part. In school mathematics education, irrational numbers arise to solve problems that the elements of the set of rational numbers cannot. Students learn the natural numbers from primary school, a knowledge which expands to a set of integers, then the rational numbers, to finally reach the set of the real numbers. The irrational numbers first introduced in school mathematics education are the solutions of the equations in the form $x^{2}-p=0$, where $p$ is a non-perfect square positive number. This research investigates what kind of knowledge do the students use when dealing with irrational numbers? Do they encapsulate these numbers into concepts or are they mostly regarded as processes? It seeks to examine the ways of thinking and the dimensions of knowledge used by a group of 157 students of different grades. The purpose of this study is to provide a record of the students' conception of irrational numbers. It explains how difficulties in the understanding of irrational numbers occur and examines whether these difficulties disappear moving up the grades. This report could be considered as

\footnotetext{
* Assistant Professor, Lebanese University, Lebanon.

${ }^{\dagger}$ Master Student, Lebanese University, Lebanon.
} 
an account of the participants' progression in the cognition, intuition and perception of irrational numbers as a part of a set of real numbers. It can be perceived as a way to describe the extent to which the participants depend on the same way of thinking when defining irrational numbers and when they have to use this definition in a different context.

\section{Backgroud}

In their ongoing study done on 46 prospective secondary mathematics teachers, Zazkis and Sirotic (2010) claimed that the minority of the wrong definitions of the irrational numbers and the heavy dependence on the calculator to decide the irrationality of these numbers is "troublesome". This study was to investigate the participants' understanding of different representations of irrational numbers, how their answers are influenced when given different representations, and about the teachers' awareness of the different definitions of the irrationals and their flexibility in using these definitions when needed. The researchers explored how the "missing link" between the two definitions of irrational numbers influenced the prospective teachers' overall understanding of irrational numbers. They showed that the same definitions used in school to define irrational numbers were also used by these prospective teachers although they have reached a high level of maturity. These results were similar to those given by Fischbein, Jehiam and Cohen (1995), that the definition of irrational numbers is not "in the active repertoire" of the participants who were high school students and prospective teachers, and who were incapable of placing the rational and irrational numbers in the appropriate set. Arcavi, Bruckheimer and Ben-Zvi (1987) also found in their study on pre-service and in-service teachers that many of the participants who had trouble in identifying rational and irrational numbers. In their study they focused on the history of the irrational number in order to develop instructional material to design courses for these teachers. Their study inspired Sirotic and Zazkis (2007a) to investigate the prospective teachers' ability to use geometric representation and the exact location of the irrational numbers as points on the number line. They found out that only $19.6 \%$ depended on the geometric origin of irrational numbers and used Pythagoras theorem to locate one of them on the number line. In order to investigate the participants' ideas about the richness of the sets of rational and irrational numbers and how they fit together in the set of real numbers, Sirotic and Zazkis (2007b) adopted the dimensions of knowledge, suggested by Tirosh et al. (1998), as a theoretical framework. They looked to see whether or not there was a conflict in the different dimensions used and detected certain inconsistencies among the three dimensions of knowledge as if the participants had opted to adjust their formal and algorithmic kinds of knowledge in order to fit their beliefs.

Voskoglou and Kosyvas (2012) conducted a study on 78 students in a public school in Greece (13-14 years) and 106 students in the Graduate Technological Educational Institute (18-19 years). They based their study on 
the hypothesis that "the main obstacle for the understanding of real numbers is the intuitive difficulties that students have with their multiple semiotic representations" and verified it. They found out that the students' understanding of rational numbers was incomplete in general, which is a great obstacle in understanding irrational numbers. They also found out that the age of the participants and their mathematical knowledge affects the comprehension of real numbers.

Deidre Arbour (2012) conducted her study on a group of thirty Canadian college-level science students, to evaluate their understanding of irrational numbers and compare the results to those found in literature. The researcher concluded that the science students faced the same obstacles that the teacher education students and high school students faced, although they take more courses in mathematics. More than half of the participants were not capable of giving a proper definition of irrational numbers and did not depend on the definition they gave in order to identify the irrational numbers.

\section{Theoretical Framework}

\section{Dimensions of Knowledge}

The three dimensions of knowledge presented by Tirosh et al. (1998) as cited by Sirotic and Zazkis (2007b) are:

- The algorithmic dimension is procedural in nature. It consists of the rules and instructions used in mathematics. The students' ability to explicate the sequential steps in different procedural operations is affected by this dimension.

- The formal dimension is about definitions of concepts, operations and theorems related to a particular content in mathematics. This dimension, as with the algorithmic one, could become highly procedural.

- The intuitive dimension includes the learners' ideas and beliefs about mathematical entities and the mental models they use for representing number concepts and operations. The intuitive knowledge could be considered as the kind of knowledge that the learners would accept directly without any need to be proved, and is preferred over other kind of explanations and solutions (Fischbein, 1987).

The researchers argue that these three dimensions are used in every mathematical activity. These dimensions overlap together so that a certain consistency between them should be acquired within the process of understanding any mathematical concept or solving any kind of problem.

\section{Modes of Thinking: Procedural and Proceptual Thinking}


Gray and Tall studied the effect of the ambiguity of natural numbers on students' ways of thinking. For example, 5 could represent the object which represents the counting of 5 elements or could be the process of the sum $2+3$. Thus, they introduced a new notion to justify the reason behind the failure and success of students in mathematics. They defined a new notion, a procept, as "the amalgam of process and concept in which process and product is represented by the same symbolism" (Gray \& Tall, 1994, p.120). This is a special kind of a concept, which is first seen as a process and then a symbol is used to represent the product of this process, resulting in giving this symbol two meanings, the process and its product. It is considered a mental activity, where the symbol acts like a shaft to switch from concentrating on process to calculating and operating, into a concept that maybe considered a teachable entity. In their study, Gray and Tall (1994) show the difference in the performance between students who use procedural thinking and proceptual thinking. The procedural thinkers focus on the procedure and the physical or the quasi-physical aids which support it whereas proceptual thinkers have the ability to manipulate the symbolism flexibly as a process or concept, freely interchanging different symbolisms for the same object (p.132).

\section{Methodology}

\section{Research Setting}

This report is a part of a study of 157 participants, in a Lebanese public high school, of three different grades ${ }^{1}$ : grade 9 (G9), grade 10 (G10) and grade 12 (G12), which is divided in two sections: life science (LS) and general sciences (GS), distributed proportionally to the number of students registered at these grades in the chosen high school. All the participants take five hours of mathematics except for the GS ones who take ten. In the Lebanese curriculum, the definition of irrational numbers is given for the first time in grade 9. They are defined as numbers that are not rational that is to say cannot be written in the form of a fraction of two integer numbers where the denominator is not equal to zero, or as the decimal numbers with an unlimited and un-periodic decimal part. At this grade, the students learn how to perform operations on irrational numbers, to compare and locate them. Afterwards in grade 10, while defining the notion of a set, irrational numbers are defined as the set of numbers that are not rational and yet are found in the set of real numbers. At this grade level, there is a problem of constructing a segment of length $\sqrt{3}$. Then the irrational numbers are used in solving equations, inequalities, performing operations, ordering real numbers... without mentioning their nature. Accordingly, the participants for this study were chosen from grades 9

\footnotetext{
${ }^{1}$ Grade 9 is the pre-secondary year, where the students apply for the official exams. Grade 10 is the first secondary year and grade 12 is the last year of secondary learning before university.
} 
and 10. Participants from G12 were chosen for being the last grade in high school before university, with the GS section for the students who have chosen to study mathematics intensively. The results from participants in G12 were also used to investigate the (in)dependency between the performance of the participants and their different grade.

The following bar graph shows the distribution of the participants according to their grades.

Figure 1. The Distribution of the Participants

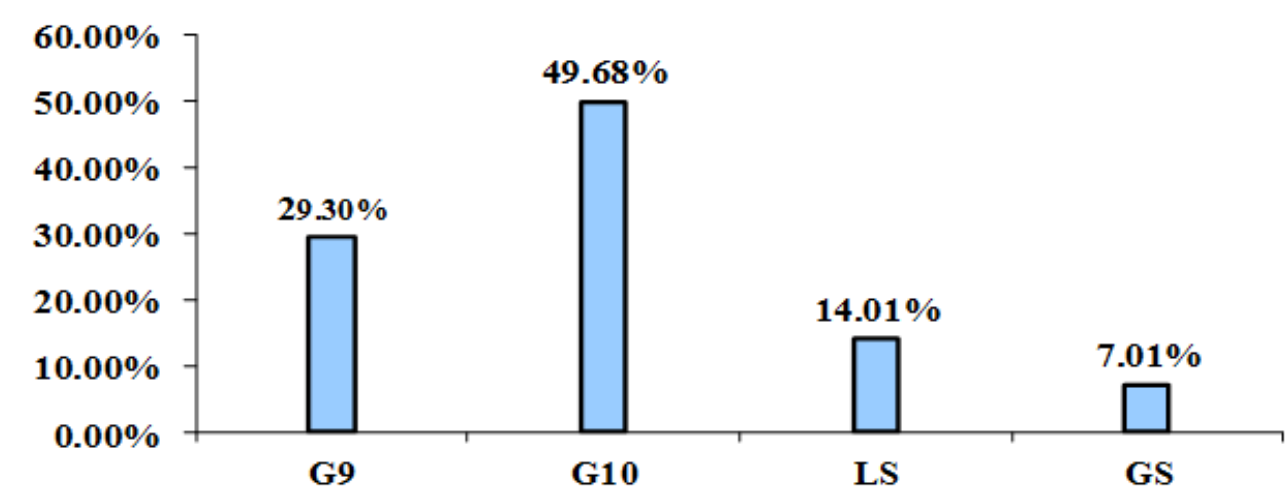

\section{Research Questions}

The proposed research questions are:

- What dimensions of knowledge do the participants use to define irrational numbers?

- Do they use the same dimension to decide the placement of these numbers within a set of irrationals?

- How do the participants conceptualize irrational numbers? What ways of thinking do they use?

- Is there a change in their performance within different grades?

\section{The Tasks}

In order to answer the research questions the following tasks were used:

- Task 1: Give the definition of an irrational number using your own words (Fischbein, Jehiam, \& Cohen, 1995).

- Task 2: Specify whether each of the following numbers is a rational or an irrational number. How do you know?

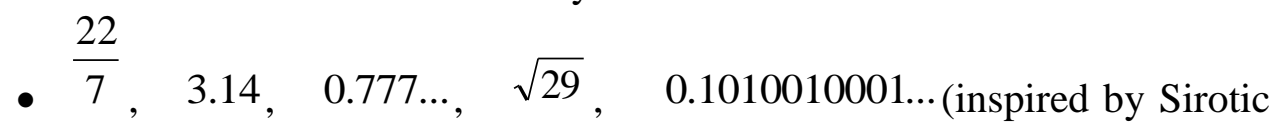


and Zazkis, 2010) , $\quad \sqrt{49}-1, \quad \frac{\sqrt{18}}{\sqrt{2}}, \frac{\sqrt{13}}{3}, \frac{1}{17}$

- Task 3: Do you think the number $\sqrt{5}$ has an exact location on a number line? If your answer is NO explain it using your own words. If your answer is YES locate this number on the number line and write your explanation of the given steps (inspired by Arcavi, Bruckheimer, \& Ben-Zvi, 1987).

\section{Data Collection}

A questionnaire, including the above mentioned tasks and a note that a calculator was allowed to be used, was distributed to the 157 participants at the same time. The importance of the accuracy of the results was explained. The privacy of the participants was taken into consideration by giving them codes instead of using their names. A pilot study was conducted a few months beforehand on a group of 30 students which led to a few changes in the wording of the tasks.

\section{Data Analysis}

The results given by the participants were analyzed using quantitative statistics and qualitative data analysis using the above mentioned theoretical frameworks.

Quantitative Data Analysis. To analyze the data using descriptive statistics, SPSS is used to find the distribution of the participants along the classified categories of each of the tasks. Using the normal distribution, Chitest is used to find out whether or not there is a change in performance within different grade levels. By comparing Sig (degree of significance) with the error ratio $(\alpha=5 \%)$, the two studied variables are said to be independent when Sig $>\alpha$, interpreted as there is no change in the distribution of results within different grades.

Qualitative Data Analysis. To seek the dimensions of knowledge and ways of thinking used by the participants, their answers to each of the given tasks are analyzed and classified as mentioned later in the research.

\section{Numerical Results}

In the following section, the ways used to classify the participants in different categories is presented, their distribution according to these categories and the results of testing the hypotheses mentioned previously.

\section{Task 1}

The distribution of the participants according to different categories. The 
responses to task 1 were classified into three main categories: True, false and no answer. The first two categories are also divided into subcategories:

- True: 1- using the non-fraction representation: for example "a number that cannot be written as a ratio of two integers" a definition given by participants in G9 and considered to be true even though the participant missed the non-zero part of the denominator, 2- True (decimal) using the decimal representation, "a number with an unlimited, non repeating decimal part".

- False: 1. false (do not end) for those who missed the un-periodicity of the irrational numbers, an "irrational number is a number that has infinite numbers after the decimal" example from a GS participant. 2. false (fraction) for those who gave the definition of the rational, "is a number that can be written as a fraction" a G10 participant answered. 3. false (decimals) for those who considered all the decimals to be irrational "is any number that has a decimal such as 1.02" answer given by an LS participant, 4.- false (confusing) for those whose definition are not clear, for example: "is a decimal number, gives many same numbers after the point for example, 5.6666..., irrational numbers are $0,3,6,9$, ..." another participant in LS answered, or "irrational number is a number either a natural number or a decimal number or a digit number that can't be simplified and has a repeating digit" answered a participant in G10. Among the confusing answers, there were answers giving the definition of prime numbers, repeated more frequently in grades 9 and 10 .

The answers were divided into many different categories in order to have more evidence on the way of thinking and different dimensions of knowledge used when defining and identifying irrational numbers.

The table below shows the percentage of the participants according to the different given categories:

Table 1. The Distribution of the Participants, for Task 1, According to the Different Subcategories

\begin{tabular}{|l|c|c|}
\hline & Frequency & Percentage \\
\hline False (Do not end) & 48 & 30.57 \\
\hline False (Confusing) & 35 & 22.29 \\
\hline True & 21 & 13.38 \\
\hline False (Fractions) & 21 & 13.38 \\
\hline True (Decimals) & 17 & 10.83 \\
\hline False (All decimals) & 9 & 5.73 \\
\hline No answer & 6 & 3.82 \\
\hline Total & 157 & 100.0 \\
\hline
\end{tabular}

The frequency of the total true answers is 38 participants out of the 157 , whereas that of the participants who gave a false response is 113 . The highest percentage goes to the participants who defined the irrational numbers as 
decimal numbers with unlimited decimal parts, thus missing the un-periodicity of this part. The results show that $72 \%$ of the participants were not capable of using their formal dimension of knowledge to define the irrational numbers properly. Later on in this research, each of the participants' performance in identifying irrational numbers and locating $\sqrt{5}$ on a number line would be compared to the definition (s)he gave in order to have a better insight whether or not giving a proper definition effects the other tasks.

The distribution of the participants according to their grade levels and their answers given to task 1 . The participants' responses are classified according to their grades; the seven different categories and the results are presented in the following table.

Table 2. The Distribution of the Participants According to their Grades and the Categories in Task 1

\begin{tabular}{|c|c|c|c|c|c|c|}
\hline & & G9 & G10 & LS & GS & Total \\
\hline \multirow[b]{2}{*}{ No answer } & Count & 3 & 3 & 0 & 0 & 6 \\
\hline & $\%$ within grade & 6.50 & 3.80 & 0.00 & 0.00 & 3.80 \\
\hline \multirow[b]{2}{*}{ True } & Count & 15 & 5 & 0 & 1 & 21 \\
\hline & $\%$ within grade & 32.60 & 6.40 & 0.00 & 9.10 & 13.40 \\
\hline \multirow[b]{2}{*}{ False } & Count & 8 & 27 & 0 & 0 & 35 \\
\hline & $\%$ within grade & 17.40 & 34.60 & 0.00 & 0.00 & 22.30 \\
\hline \multirow[b]{2}{*}{ False (Do not end) } & Count & 10 & 19 & 14 & 5 & 48 \\
\hline & $\%$ within grade & 21.70 & 24.40 & 63.60 & 45.50 & 30.60 \\
\hline \multirow[b]{2}{*}{ False (All decimals) } & Count & 2 & 4 & 3 & 0 & 9 \\
\hline & $\%$ within grade & 4.30 & 5.10 & 13.60 & 0.00 & 5.70 \\
\hline \multirow[b]{2}{*}{ False (Fractions) } & Count & 3 & 15 & 1 & 2 & 21 \\
\hline & $\%$ within grade & 6.50 & 19.20 & 4.50 & 18.20 & 13.40 \\
\hline \multirow[b]{2}{*}{ True (Decimals) } & Count & 5 & 5 & 4 & 3 & 17 \\
\hline & $\%$ within grade & 10.90 & 6.40 & 18.20 & 27.30 & 10.80 \\
\hline \multirow{2}{*}{ Total } & Count & 46 & 78 & 22 & 11 & 157 \\
\hline & $\%$ within grade & 100.00 & 100.00 & 100.00 & 100.00 & 100.00 \\
\hline
\end{tabular}

Chi-square calculated $=58.341, \mathrm{df}=18, \mathrm{Sig}=0.000<\alpha$, then the variables are not independent

Sig $=0.000<\alpha$ then the variables are not independent, and there is a relation between the grade of the participants and their answers to this task. As the table shows, G9 participants took the highest percentage for giving a correct definition using the non-fraction representation of irrational numbers. The fact that this definition is taught at this grade is clearly a factor here, which would have affected the results. This table shows that only 4 GS participants gave a correct definition, one using the non-fraction representation of irrational numbers and the rest depending on the decimal one.

Task 2 
The distribution of the participants according to the categories of task 2. The answers given by the participants to each of the numbers found in task 2 are classified into three categories: No answer (when no answer was given), True (when the participant places the number in the correct set of numbers), and False (when the participant places the number in the incorrect set of numbers).

In the absence of interviews, the numbers in this task were chosen in order to have a better idea of what kind of criteria was used to identify the numbers. For example, to eliminate the factor that the participants may consider $\frac{22}{7}$ to be irrational since it is the rational approximation of $\pi, \frac{1}{17}$ was added so that the reason behind the answer could clarified. Thus, participants considering these two rational fractions as irrational numbers were considered to have depended on the decimal representation of these numbers to place them in the correct set of numbers and then compared to the definition given in task 1 . In other words, giving a correct definition of irrational numbers, using the non-fraction representation of these numbers, and then considering these two fractions to be irrational numbers indicates inconsistency in using the formal and the intuitive dimension of knowledge.

Additionally, for the participants who might consider all the numbers under the radical to be irrational, $\sqrt{49}-1$ and $\frac{\sqrt{18}}{\sqrt{2}}$ which are two natural numbers, written in a different representation, were added to this task. Not performing the two processes found in these two numbers is considered as an indication of thinking of these numbers as objects, thus there is an incapability of switching easily between the process and the object found in each number.

Moreover, $\frac{\sqrt{13}}{3}$, an irrational number written in the form of a fraction, was used for the participants who would miss the fact that to be a rational number, both the numerator and the denominator should be integers. Number $0.777 \ldots$ was included as the rational number with a periodic decimal part where $0.101001 \ldots$ is an irrational number with a pattern in its decimal part. These two numbers were included in this task in order to determine the percentage of the participants who could differentiate between them and to consider those who realized the nature of both as consistent in deploying the definition of the irrational numbers using the decimal representation. Defining irrational numbers to be unlimited and not repeating decimal numbers then placing $0.777 \ldots$ in this set of numbers is considered as inconsistency in using dimensions of knowledge.

Below is the table showing the distribution of the participants' answers between the three different categories of this task. 
Table 3. The Distribution of the Participants According to the Categories of Task 2

\begin{tabular}{|l|c|c|c|}
\hline & No answer & True & False \\
\hline$\frac{22}{7}$ & $5.73 \%$ & $17.20 \%$ & $\mathbf{7 7 . 0 7 \%}$ \\
\hline $\mathbf{3 . 1 4}$ & $10.83 \%$ & $\mathbf{5 6 . 0 5 \%}$ & $33.12 \%$ \\
\hline $\mathbf{0 . 7 7 7} \ldots$ & $13.38 \%$ & $26.75 \%$ & $\mathbf{5 9 . 8 7 \%}$ \\
\hline$\sqrt{29}$ & $15.92 \%$ & $\mathbf{6 9 . 4 3 \%}$ & $14.65 \%$ \\
\hline $\mathbf{0 . 1 0 1 0 0 1 0 0 0 1 0 0 0 0 1 \ldots}$ & $8.28 \%$ & $\mathbf{7 0 . 7 0 \%}$ & $21.02 \%$ \\
\hline$\sqrt{49}-1$ & $8.28 \%$ & $\mathbf{7 9 . 6 2 \%}$ & $12.10 \%$ \\
\hline$\frac{\sqrt{18}}{\sqrt{2}}$ & $9.55 \%$ & $\mathbf{7 7 . 0 7 \%}$ & $13.38 \%$ \\
\hline$\frac{\sqrt{13}}{3}$ & $12.10 \%$ & $\mathbf{7 5 . 8 0 \%}$ & $12.10 \%$ \\
\hline$\frac{1}{17}$ & $11.46 \%$ & $15.29 \%$ & $\mathbf{7 3 . 2 5 \%}$ \\
\hline
\end{tabular}

The remarkably high percentage of correct classifications for $\sqrt{49}-1$ and $\frac{\sqrt{18}}{\sqrt{2}}$ may indicate the preference of the participants to perform processes, or that they are acquainted with these numbers in their mathematics curriculum. However, the high percentage of the participants who considered the two given rational fractions, with long periods, to be irrational numbers proved the participants relief to change to the decimal representation, and perform the process found in these numbers before placing them in the convenient set of numbers. More than half the participants recognized 3.14 as a rational number, however $56.05 \%$ of true answers are considered to be a low percentage. In the next table, the distribution of the answers to this number within different grades is shown.

The high percentage of the correct classification of $0.101001 \ldots$ as an irrational number lead to an investigation to better understand the reasons behind this high percentage. Since no interviews were conducted, a relation between the answers given to this number and to $0.777 \ldots$ and the results were as follows: 83 participants $(52.87 \%)$ considered them both irrational thus missing the un-periodic condition for the decimal part of irrational numbers, 9 participants $(5.73 \%)$ relied on their definition that every decimal is irrational, 19 participants $(12.1 \%)$ differentiated between the two numbers but only one of them recognized the two rational fractions.

The high percentage of the participants identifying $\frac{\sqrt{13}}{3}$ to be an irrational number could be justified by the fact that the irrational numbers were introduced to these participants for the first time as square roots on non-perfect positive numbers. 
The distribution of the participants according to their grades and their answers given to task 2. For each of the given numbers, the percentage of the participants within each category and each grade is shown in the following table, in addition to the calculated Sig.

Table 4. The Distribution of the Participants According To Their Grades and Their Classification of the Numbers Given In Task 2

\begin{tabular}{|c|c|c|c|c|c|c|}
\hline & & G9 & G10 & LS & GS & Sig \\
\hline \multirow{3}{*}{$\frac{22}{7}$} & No Answer & $8.70 \%$ & $2.60 \%$ & $13.60 \%$ & $0.00 \%$ & \multirow{3}{*}{0.018} \\
\hline & True & $21.70 \%$ & $10.30 \%$ & $18.20 \%$ & $45.50 \%$ & \\
\hline & False & $69.60 \%$ & $87.20 \%$ & $68.20 \%$ & $54.50 \%$ & \\
\hline \multirow{3}{*}{3.14} & No Answer & $15.20 \%$ & $6.40 \%$ & $22.70 \%$ & $0.00 \%$ & \multirow{3}{*}{0.002} \\
\hline & True & $34.80 \%$ & $62.80 \%$ & $59.10 \%$ & $90.90 \%$ & \\
\hline & False & $50.00 \%$ & $30.80 \%$ & $18.20 \%$ & $9.10 \%$ & \\
\hline \multirow{3}{*}{$0.777 \ldots$} & No Answer & $10.90 \%$ & $17.90 \%$ & $9.10 \%$ & $0.00 \%$ & \multirow{3}{*}{0.388} \\
\hline & True & $23.90 \%$ & $23.10 \%$ & $36.40 \%$ & $45.50 \%$ & \\
\hline & False & $65.20 \%$ & $59.00 \%$ & $54.50 \%$ & $54.50 \%$ & \\
\hline \multirow{3}{*}{$\sqrt{29}$} & No Answer & $17.40 \%$ & $15.40 \%$ & $22.70 \%$ & $0.00 \%$ & \multirow{3}{*}{0.765} \\
\hline & True & $67.40 \%$ & $69.20 \%$ & $68.20 \%$ & $81.80 \%$ & \\
\hline & False & $15.20 \%$ & $15.40 \%$ & $9.10 \%$ & $18.20 \%$ & \\
\hline \multirow{3}{*}{$0.101001 \ldots$} & No Answer & $8.70 \%$ & $7.70 \%$ & $13.60 \%$ & $0.00 \%$ & \multirow{3}{*}{0.562} \\
\hline & True & $65.20 \%$ & $75.60 \%$ & $68.20 \%$ & $63.60 \%$ & \\
\hline & False & $26.10 \%$ & $16.70 \%$ & $18.20 \%$ & $36.40 \%$ & \\
\hline \multirow{3}{*}{$\sqrt{49}-1$} & No Answer & $8.70 \%$ & $6.40 \%$ & $18.20 \%$ & $0.00 \%$ & \multirow{3}{*}{0.384} \\
\hline & True & $78.30 \%$ & $79.50 \%$ & $72.70 \%$ & $100.00 \%$ & \\
\hline & False & $13.00 \%$ & $14.10 \%$ & $9.10 \%$ & $0.00 \%$ & \\
\hline \multirow{3}{*}{$\frac{\sqrt{18}}{\sqrt{2}}$} & No Answer & $10.90 \%$ & $6.40 \%$ & $22.70 \%$ & $0.00 \%$ & \multirow{3}{*}{0.341} \\
\hline & True & $76.10 \%$ & $79.50 \%$ & $68.20 \%$ & $81.80 \%$ & \\
\hline & False & $13.00 \%$ & $14.10 \%$ & $9.10 \%$ & $18.20 \%$ & \\
\hline \multirow{3}{*}{$\frac{\sqrt{13}}{3}$} & No Answer & $17.40 \%$ & $9.00 \%$ & $18.20 \%$ & $0.00 \%$ & \multirow{3}{*}{0.576} \\
\hline & True & $69.60 \%$ & $79.50 \%$ & $68.20 \%$ & $90.90 \%$ & \\
\hline & False & $13.00 \%$ & $11.50 \%$ & $13.60 \%$ & $9.10 \%$ & \\
\hline \multirow{3}{*}{$\frac{1}{17}$} & No Answer & $13.00 \%$ & $10.30 \%$ & $18.20 \%$ & $0.00 \%$ & \multirow{3}{*}{0.105} \\
\hline & True & $15.20 \%$ & $11.50 \%$ & $13.60 \%$ & $45.50 \%$ & \\
\hline & False & $71.70 \%$ & $78.20 \%$ & $68.20 \%$ & $\mathbf{5 4 . 5 0 \%}$ & \\
\hline
\end{tabular}

The calculation of Sig shows that the two variables, the classification of the numbers and the grades are only not independent in two cases: $\frac{22}{7}$ and 3.14. As for the other numbers, the results verify that the two variables are independent, and that the responses of the participants were not related to their grade. 
The number 3.14 is used for a long time in school learning as the decimal replacement for $\pi$. This replacement had its effects on the participants in G9, where the highest percentage of those who considered this number to be irrational goes for them. However, the percentage of false answers decreased with higher grades to reach only $9.1 \%$ in GS.

Task 3. The answers of the participants are classified into four categories: true, false, false (do not have exact location), false (by approximation) and no answer. Representing irrational numbers like $\sqrt{2}, \sqrt{3}, \sqrt{5} \ldots$ is found in the mathematics textbook for grade 9 that is used by the participants. Thus, the aim of this task was to examine participants' knowledge about the exact location of the irrational numbers on the number line and their ability to locate one of these numbers.

True: participants who answered yes to the first part of the task even if they did not locate the number. They answered intuitively that it could be located but were not capable of answering algorithmically. For example a participant in G9 answered: "yes it has an exact location but I don't know how". Only one participant in GS was capable of locating this number correctly, using the metric relations in a right triangle. She drew a circle, of diameter 6 units, one unit below the number line and 5 units above, and perpendicular to it. This circle intersects the number line in two points, thus the one with positive abscissa is $\sqrt{5}$.

False: for the participants who considered changing the unit of the number line into multiples of $\sqrt{5}$, for example one participant in G10 said: "this could be done by changing the unit of the number line to $\sqrt{5}, 2 \sqrt{5} \ldots$. . This

False (do not have exact location): is the group of the participants who answered that such a number does not have an exact location on the number line because "it has not a limited value there are many numbers after the decimal" as participant number 14 in LS wrote, or because "it is endless" written by participant number 6 in G9.

False (by approximation): this group considered that it can only be constructed after being approximated. For example: "yes, $\sqrt{5}=2.23=2$ " or another approximation " $\sqrt{5}=2.25 "$.

No answer: the group of participants who gave no answer. 
The distribution of the participants according to different categories in task 3

Figure 3. The Distribution of the Percentage of the Participants According to their Answers to the Second Part in Task 3

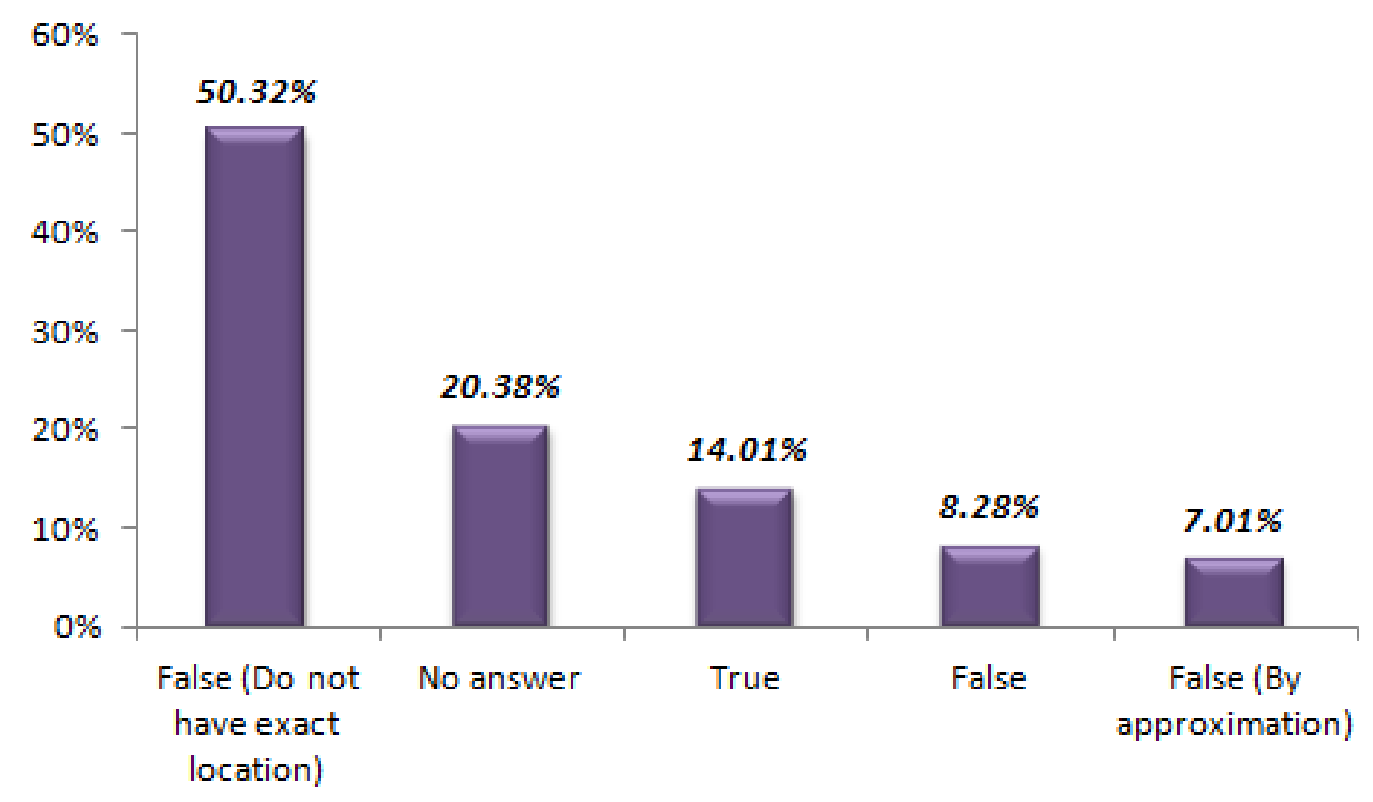

Total false $=65.61 \%$

The distribution of the participants' answers in task 3 according to their grade. The following table shows the frequencies and the percentages of the participants giving different responses, as categorized in this task.

Table 5. The Distribution of the Participants According to their Grades and the Categories in Task 3

\begin{tabular}{|c|c|c|c|c|c|c|}
\hline & & G9 & G10 & $\mathbf{L S}$ & GS & Total \\
\hline \multirow{2}{*}{ No answer } & Count & 10 & 16 & 6 & 1 & 33 \\
\hline & $\%$ within Class & 21.70 & 20.50 & 27.30 & 9.10 & 21.00 \\
\hline \multirow{2}{*}{ True } & Count & 7 & 11 & 2 & 1 & 21 \\
\hline & $\%$ within Class & 15.20 & 14.10 & 9.10 & 9.10 & 13.40 \\
\hline \multirow{2}{*}{ False } & Count & 0 & 0 & 0 & 1 & 1 \\
\hline & $\%$ within Class & 0.00 & 0.00 & 0.00 & 9.10 & 0.60 \\
\hline \multirow{2}{*}{ False (By approximation) } & Count & 3 & 7 & 1 & 1 & 12 \\
\hline & $\%$ within Class & 6.50 & 9.00 & 4.50 & 9.10 & 7.60 \\
\hline \multirow{2}{*}{$\begin{array}{l}\text { False (Do not have exact } \\
\text { location) }\end{array}$} & Count & 26 & 44 & 13 & 7 & 90 \\
\hline & $\%$ within Class & 56.50 & 56.40 & 59.10 & 63.60 & 57.30 \\
\hline \multirow[b]{2}{*}{ Total } & Count & 46 & 78 & 22 & 11 & 157 \\
\hline & $\%$ within Class & $\begin{array}{c}100.0 \\
0\end{array}$ & $\begin{array}{c}100.0 \\
0\end{array}$ & $\begin{array}{c}100.0 \\
0\end{array}$ & $\begin{array}{c}100.0 \\
0\end{array}$ & $\begin{array}{c}100.0 \\
0\end{array}$ \\
\hline
\end{tabular}

Chi-square calculated $=15.716, \mathrm{df}=12, \mathrm{Sig}=0.205>\alpha$, then the variables are independent 
The calculated $\operatorname{Sig}=0.000<\alpha$, then the two variables are dependent and there is a relation between the grade and the ability of the participants to locate the number $\sqrt{5}$. The highest percentage in all grades is for the category False (Do not have exact location), indicating that more than half the participants in each grade believe that this number could not be located exactly on the number line, because it is an irrational number.

\section{Qualitative Analysis Based on the Chosen Theoretical Frameworks}

The participants' responses are analyzed in a search for the dimensions of knowledge and for the possible ways of thinking about irrational numbers that could be used when answering the given tasks. Also an inquiry into indications of consistency between the dimensions of knowledge and for the conceptualization of the irrational numbers is conducted. The analysis is divided into two main sub-categories: consistencies and inconsistencies between the dimensions of knowledge used, and the conceptualization of irrational numbers as processes or objects.

\section{According to the Dimensions of Knowledge}

Consistency in using the formal dimension. The participants who gave a correct definition in task 1 and used this definition in task 2 are considered to be consistent in using the same formal dimension of knowledge. Taking into consideration their answers to all the numbers given in task 2 , only 5 participants are judged to be consistent, and only one out of these five, a GS student, located the number correctly in task3. Her answer to task 1 is: "irrational numbers are numbers that can't be written as a ratio of two numbers". However, this participant could not be considered a proceptual thinker since in task 2 she considered $\frac{\sqrt{18}}{\sqrt{2}}$ to be irrational, missing the process part of this number, " $\sqrt{29}, \frac{\sqrt{18}}{\sqrt{2}}, \frac{\sqrt{13}}{3}$ are irrational numbers that can't be written as a fraction of two natural numbers". Another of the participants, in G10, gave the correct non-fraction representation of the irrational numbers and used her definition to place the numbers in task 2, yet, neither could she be considered a proceptual thinker based on the fact that she conceptualized $\sqrt{5}$ as a non-exact number that can never be located exactly, thus as a non-ending process: "No, I think $\sqrt{5}$ doesn't have an exact location on the number line $\sqrt{5}=2.236067977$.. which is endless then we take a very sensitive scale which is so difficult to be done". The other three participants, one in LS, one in G10 and the final in G9, used the decimal representation of irrational numbers and considered the two rational fractions in task 2 to be irrational when changing them to their decimal approximation shown on the calculator display. Considering them procedural thinkers is based on the fact that they changed all 
the given numbers in task 2 to their decimal form before making any decision about their nature.

Consistency in using the intuition dimension. These are the participants who relied on their understanding of the nature of irrational numbers to define them and to answer task 2. For example, the participants who defined irrational numbers as decimals that do not end, used this definition and changed every given number in task 2 to its decimal representation then considered every non terminating number to be an irrational one is placed in this group. The majority of this group was the participants who defined irrational numbers as unlimited decimal numbers (48 participants). There are 39 participants out of the 48 who used this exact definition, and 5 who managed to twist the definition they used to suit their beliefs and considered 3.14 to be an irrational number as being the decimal approximation of $\pi$. The remaining 4 believed that the answer given on the calculator display were the exact values of the numbers given in task 2 and thus categorized them all as rational numbers.

Another group of 9 participants are considered to be consistent in using their beliefs. This group considered all the decimal numbers to be irrational except for the two natural ones.

These participants performed these tasks poorly and are considered to be procedural thinkers.

Inconsistency between the formal and the intuitive dimension. They are 32 participants who preferred to use their beliefs in task 2 instead of the formal knowledge they used in task 1 . For example, they would depend on the nonfraction definition of an irrational number and then consider $\frac{22}{7}$ and $\frac{1}{17}$ to be irrational. Or, they have considered the irrational numbers to not repeat but place $0.777 \ldots$ as irrational. To be in this group, the definition to task 1 should have been considered true.

These participants are divided into two groups; the group that thought of the numbers as processes by writing its decimal representation next to each number, and the group that thought of the given numbers as constant objects having certain forms, and these forms are the criteria for defining irrational numbers. Neither one of these participants had the capability to switch between the processes found in the numbers nor the product of these processes in order to place these numbers in their correct set of numbers, and thus are considered to have a procedural thinking of numbers in general.

Numbers as a process. Of those who used the non-fraction definition of the irrational numbers, there are 18 participants who, in task 2 , used the decimal representation of the numbers. One of the participants in G9 defined irrational numbers as: "numbers that not expressible as a ratio of two integers, and having an infinite and non recurring expressed as a decimal", and in task 2: 
$\frac{22}{7}, 3.14, \sqrt{29}, 0.101001 \ldots, \frac{\sqrt{13}}{3}, \frac{1}{17}$ are irrational numbers because it is a decimal". Another group of 13 participants gave the correct decimal definition of the irrational numbers and did not use it to recognize $0.777 \ldots$ as rational.

Numbers as object. One participant is considered to depend heavily on the given representation of the numbers. This participant is in G10 and depended on the non-fraction definition that he gave in task 1 , thus considered all the numbers that are written as a fraction to be rational, i.e. $\frac{\sqrt{13}}{3}$ : rational numbers, as for $3.14,0.777 \ldots$, and $\sqrt{49}-1$ : irrational. This participant did not account for the nature of the numerator and denominator of rational numbers.

Mixed dimensions of knowledge. This is the group of 56 participants who are considered to be incapable of identifying a certain image of the irrational numbers. These participants either give the definition of prime numbers yet consider decimal numbers to be irrational, or give the definition of irreducible fractions and then consider $\pi$ as irrational. Some of these participants gave definitions that were mixed between prime numbers and rational fractions. For example: "a fraction that cannot be simplified anymore", "a fraction that when divided gives an endless decimal number", "are numbers that cannot be divided", "are numbers that cannot be simplified by any number else except one and itself".

Irrational numbers as never ending process. The procedural way of thinking is detected among 103 participants who thought of $\sqrt{5}$ as a nonending process and thus could not have an exact location on the number line. Having to approximate the irrational number before locating it on the number line is a common misconception in this research, similar to conclusions drawn by Sirotic and Zazkis (2007a). Gary and Tall (1994) consider that the confusion about the nature of infinity will slow down the students' development of the procept notion of irrational numbers. The concept of infinity is not introduced at grade levels 9 or 10, however, many participants at these grades depend on their intuitive knowledge of this concept and used it as an explanation to why an irrational number cannot have an exact location on the number line. Here is a sample of answers given: a participant in GS answered: "no because it is irrational", another one : "no, since it is an endless number, so if you located in such a place it always can be shifted by one unit", a participant in LS answered: "no because it has infinite numbers after the decimal", another one in G10: "No location on the number line because this kind of numbers can't finish and can't have an exact value", and one in G9: "no because it is an infinite number and infinite numbers cannot be drawn on the number line". This last justification raises the question about the language used in the classroom when dealing with irrational numbers. 


\section{Conclusion}

At the outset of the research, the main purpose was to understand more deeply what an irrational number is for the students, process or object or procept, and to have a better insight of their conceptualization the three tasks were used along with mixed methods to analyze their results. The analysis of the answers showed that there is a significant change in the participants' performance in tasks 1 and 3 and when classifying the two numbers $\frac{22}{7}$ and 3.14 .

Moreover, 24.2\% of the participants gave a proper definition of the irrational numbers and $14.01 \%$ considered $\sqrt{5}$ could be located. As for task 2 a heavy dependence on the decimal representation of all the given numbers was noticed, with inconsistency in using the dimension of knowledge when defining irrational numbers and identifying these numbers. The results show that most of the participants prefer what they believe the irrational numbers are to the formal words that they may use when defining these numbers. Only five participants out of the 157 were consistent in using the formal dimension in the first two tasks, 57 were consistent in depending on their beliefs when answering both tasks 1 and 2, whereas 56 participants were classified as mixed dimensions users since they were considered to not have a clear image of what an irrational number could be.

According to the participants' responses, it was claimed in this research, that the way of thinking used regarding irrational numbers (most probably about real numbers) is mostly procedural, where these numbers are a never ending processes. There are 103 participants who insisted that an irrational number "doesn't end" and thus cannot be constructed exactly, this included $63.6 \%$ of the GS participants who are considered to be the best performers in mathematical thinking in this high school.

The fact that irrational numbers are difficult in their nature forms a major obstacle in conceptualizing these numbers as procepts. Switching from the process notion to the procept one is a very delicate procedure that helps to better deal with real numbers in general and the irrational ones in particular. This switch could be considered as essential to the understanding of the set of real numbers, and it is not achieved by the participants in this research. The conceptualization of irrational numbers would help the learners understand the completeness of the set of real numbers thus affecting their conceptualization of the continuity of a function and the concept of its limit. We believe that this necessary switch could be achieved by showing the equivalence of the two definitions of the irrational numbers in addition to their geometric representation. Such equivalence may lead to helping students conceive the fact that the irrational numbers are constant and static objects even when they have an unlimited un-periodic decimal part. The two definitions used together and the focus on the ability to locate certain irrational numbers and drawing them as segments with exact lengths, in addition to exposing the origin of these 
numbers, would help learners in encapsulating the irrational numbers into concepts and thinking proceptually when facing situations including these numbers.

It is also recommended that further investigations are conducted into teachers' approaches to dealing with irrational numbers in the classroom and into extent to which the language and teaching methods used affect the learners' conceptualization of these numbers. In addition, there is a need for an inquiry into the curriculums' objectives concerning irrational numbers and the focus on assuring the proceptual thinking of these numbers.

\section{References}

Arbour, D. (2012). Students' Understanding of Real, Rational and Irrational Number (Master thesis). Concoria University, Quebec, Canada. Retrieved from bit.ly/1 Kbj2xL.

Arcavi, A. Bruckheimer, M. \& Ben-Zvi, R. (1987). History of mathematics for teachers: the case of irrational numbers. For the Learning of Mathematics, $7(2), 18-23$.

Dedekind, R. (1901). Essays of the Theory of Numbers. Beman, W. W. (Trans.) Chicago, IL: The Open Court Publishing Company.

Fischbein, E. (1987). Intuition in science and mathematics: an educational approach. Dordrecht, The Netherlands: Reidel.

Fischbein, E., Jehiam, R. \& Cohen, D. (1995). The concept of irrational number in high-school students and prospective teachers. Educational Studies in Mathematics, 29, 29-44.

Gray, E., \& Tall, D. (1994). Duality, ambiguity and flexibility: A proceptual view of simple arithmetic. Journal for Research in Mathematics Education, 26(2), 115-141.

Sirotic, N. \& Zazkis, R. (2007a). Irrational numbers: The gap between formal and intuitive knowledge. Educational Studies in Mathematics, 65(1), 49-76.

Sirotic, N. \& Zazkis, R. (2007b). Irrational numbers on the number line - where are they? International Journal of Mathematical Education in Science and Technology, 38(4), 477-488.

Tirosh, D., Fischbein, E., Graeber, A., \& Wilson, J. (1998). Prospective elementary teachers' conceptions of rational numbers. Retrieved June 2003 from bit.ly/1Pkp9yI.

Voskoglou, M.G., \& Kosyvas, G.D. (2012). Analyzing students' difficulties in understanding real numbers. Journal of Research in Mathematics Education, 1(3), $301-336$.

Zazkis, R. \& Sirotic, N. (2010). Representing and defining irrational numbers: exposing the missing link. CBMS Issues in Mathematics Education, 16. 